\title{
WEAK CONVERGENCE OF MEASURES IN CONSERVATIVE SYSTEMS
}

\author{
V. V. Kozlov and D. V. Treschev
}

UDC 517.19

Families of probability measures on the phase space of a dynamical system are considered. These measures are obtained as shifts of a given measure by the phase flow. Sufficient conditions for the existence of the weak convergence of the measures as the rate of the shift tends to infinity are suggested. The existence of such a limit leads to a new interpretation of the second law of thermodynamics. Bibliography: 5 titles.

\section{INTRODUCTION}

Let $\Gamma$ be the phase space of the dynamical system generated by the differential equation

$$
\dot{x}=u(x), \quad x \in \Gamma .
$$

We assume that any solution of this system can be continued to the whole time axis. Then the corresponding phase flow $g^{t}$ is a well-defined one-parameter group of diffeomorphisms of the space $\Gamma$.

Following Gibbs [1], we consider, at the initial time moment $t=0$, a probability measure $\mu$ in $\Gamma(\mu(\Gamma)=1)$. The flow $g^{t}$ shifts the measure $\mu$ : we set $\mu_{t}=g^{t *}(\mu)$, where

$$
g^{t *}(\mu)(D)=\mu\left(g^{-t}(D)\right) \quad \text { for any } \mu \text {-measurable set } \quad D \subset \Gamma .
$$

Gibbs tried to show that as $t \rightarrow \infty$, the measures $\mu_{t}$ tend (in some sense) to a stationary measure, which corresponds to a heat equilibrium state. This was a motivation for introducing in Hamiltonian systems the microcanonical probability distribution, whose density depends only on the energy.

We study the problem of the weak convergence of the measures $\mu_{t}$. This is quite natural from the viewpoint of the justification of thermodynamics - the transition to the macroscopic description of the evolution of a dynamical system.

We assume that the system (1.1) preserves a measure $\nu$ on $\Gamma$ and the measure $\mu$ has a density $\rho \in L_{2}(\Gamma, \nu)$, i.e., $d \mu=\rho d \nu$. This allows us to determine the mean values for functions from the space $L_{2}(\Gamma, \nu)$.

Since the measure $\nu$ is invariant, the density $\rho_{t}$ of the measure $\mu_{t}$ has the form

$$
\rho_{t}(x)=\rho \circ g^{-t}(x)
$$

Given a measure $\bar{\mu}$ with density $\bar{\rho} \in L_{2}(\Gamma, \nu)$, the measures $\mu_{t}$ are said to converge weakly to $\bar{\mu}($ as $t \rightarrow \infty)$ if for any $\varphi \in L_{2}(\Gamma, \nu)$, the function

$$
K(t)=\left(\rho \circ g^{-t}, \varphi\right)=\int_{\Gamma} \varphi(x) \rho \circ g^{-t}(x) d \nu(x)
$$

converges to $\int_{\Gamma} \varphi \bar{\rho} d \nu$.

Below we present sufficient conditions for weak convergence and describe the limit measures.

The paper is organized as follows. In Sec. 2, assuming that the weak limit exists, we present a formula for the limit measure. In Sec. 3, we introduce the class of systems (systems (3.1)) we are going to deal with. We present basic motivations and examples and discuss the main technical tool used in the proofs - a generalization of the von Neumann ergodic theorem where the time average is replaced by the average with respect to some probability measure. In Sec. 4, we specify the class of systems (so-called foliated flows) for which we prove the weak convergence of the measures $\mu_{t}$. Typical systems from this class are geodesic flows and other Hamiltonian quasi-homogeneous systems. Proofs of the main results are given in Sec. 5. Finally, in Sec. 6 we prove that the entropy of the limit measure $\bar{\mu}$ is not less than the entropy of the original measure $\mu$.

Published in Zapiski Nauchnykh Seminarov POMI, Vol. 300, 2003, pp. 194-205. Original article submitted November 30, 2002. 


\section{WEAK LIMIT}

Theorem 1. Assume that for a function $\varphi \in L_{2}(\Gamma, \nu)$, there exists the limit

$$
\lim _{t \rightarrow \infty} K(t)=K_{\infty}
$$

Then $K_{\infty}=(\bar{\rho}, \varphi)$, where

$$
\bar{\rho}(x)=\lim _{T \rightarrow \infty} \frac{1}{T} \int_{0}^{T} \rho \circ g^{t}(x) d t .
$$

Relation (2.1) holds almost everywhere on $\Gamma$. Since $\rho \in L_{1}$, it follows from the Birkhoff ergodic theorem that $\bar{\rho}$ is defined almost everywhere, nonnegative, and $g^{t}$-invariant, and (if $\Gamma$ is compact)

$$
\int_{\Gamma} \bar{\rho} d \nu=1
$$

Hence $\bar{\rho}$ is the density of a stationary probability measure.

If $\lim K(t)$ exists for any $\varphi \in L_{2}(\Gamma, \nu)$, then the function $\bar{\rho}$ satisfying the equation $K_{\infty}=(\bar{\rho}, \varphi)$ is unique. This implies the following corollary.

Corollary 2.1. If the measures $\mu_{t}$ converge weakly to $\bar{\mu}$, then the corresponding density $\bar{\rho}$ satisfies $(2.1)$.

Proof of Theorem 1 . Let $\left(\rho_{t}, \varphi\right) \rightarrow K_{\infty}$ as $t \rightarrow \infty$. Then, by the Cauchy theorem,

$$
\lim _{T \rightarrow \infty} \frac{1}{T} \int_{0}^{T}\left(\rho_{t}, \varphi\right) d t=K_{\infty}
$$

Note that the integral in the left-hand side exists for all $T$ if $\rho, \varphi \in L_{2}(\Gamma, \nu)$ (see, for example, [4]). By the Fubini theorem,

$$
\frac{1}{T} \int_{0}^{T}\left(\rho_{t}, \varphi\right) d t=\int_{\Gamma} \widetilde{\rho}_{T}(x) \varphi(x) d \nu(x)
$$

where

$$
\widetilde{\rho}_{T}=\frac{1}{T} \int_{0}^{T} \rho \circ g^{t} d t
$$

By the von Neumann theorem, $\int_{\Gamma}(\widetilde{\rho}-\bar{\rho}) d \nu \rightarrow 0$ as $T \rightarrow \infty$. This implies that

$$
\lim _{T \rightarrow \infty} \frac{1}{T} \int_{0}^{T}\left(\rho_{t}, \varphi\right) d t=(\bar{\rho}, \varphi) .
$$

Indeed,

$$
\left[\int_{\Gamma}\left(\widetilde{\rho}_{T}-\bar{\rho}\right) \varphi d \nu\right]^{2} \leq \int_{\Gamma}\left(\widetilde{\rho}_{T}-\bar{\rho}\right)^{2} d \nu \int_{\Gamma} \varphi^{2} d \nu \rightarrow 0
$$

as $T \rightarrow \infty$.

\section{Generalization of the statistical ergodic theorem}

We consider the problem of existence of the weak limit of the densities $\rho_{t}$ for dynamical systems of the following form:

$$
\dot{z}=v(z, \omega), \quad \dot{\omega}=0 .
$$

The phase space $\Gamma$ is the cross product $\Lambda \times D$, where $\Lambda=\left\{z_{1}, \ldots, z_{n}\right\}$ is a smooth $n$-dimensional manifold and $D$ is a domain in $\mathbb{R}^{m}=\left\{\omega_{1}, \ldots, \omega_{m}\right\}$. The coordinates $\omega$ are constants of motion. We assume that for a fixed $\omega$, the system on $\Lambda$ has an invariant measure $d \nu=\lambda(z, \omega) d^{2 n} z$.

As a particular case, we obtain a Hamiltonian system. Here $m=1, \Lambda$ is an energy level, and $\omega$ is the value of the energy. The phase space $\Gamma$ of the Hamiltonian system breaks into the cells $h_{1} \leq H \leq h_{2}$, where the function $H$ has no critical values in the interval $\left(h_{1}, h_{2}\right)$.

First consider the particular case of $(3.1)$ where the field $v(z, \omega)$ is the product $\omega v(z)$, with $v(z)$ being a smooth vector field on $\Lambda$. The phase flow of this system is the family of transformations $\left\{g^{\omega t}\right\}$, where $\left\{g^{t}\right\}$ is the flow of the dynamical system

$$
\dot{z}=v(z), \quad z \in \Lambda \text {. }
$$

Its invariant measure $\nu$ does not depend on the parameter $\omega$. 
Theorem 2. Let $f_{1}$ and $f_{2}$ be functions from $L_{2}(\Lambda, \nu)$, where the measure $\nu$ is absolutely continuous with respect to the measure determined on $\Lambda$ by some Riemannian metric and $\nu(\Lambda)<\infty$; and let $h \in L_{1}(I, d \omega)$ be an integrable function on a measurable set $I \subset \mathbb{R}=\{\omega\}$.

Then

$$
\lim _{t \rightarrow \infty} \int_{I} h(\omega)\left(f_{1} \circ g^{t}, f_{2}\right) d \omega=\left(\bar{f}_{1}, f_{2}\right) \int_{I} h(\omega) d \omega
$$

Theorem 2 contains, as a particular case, the classical von Neumann theorem (see [4]). Indeed, let $h$ be the indicator of the interval $[0,1]$, i.e., $h(\omega)=1$ for $\omega \in[0,1]$ and $h(\omega)=0$ for $\omega \notin[0,1]$. Then for any integrable function $\varphi: \mathbb{R} \rightarrow \mathbb{R}$

$$
\int_{-\infty}^{+\infty} h(\omega) \varphi(\omega t) d \omega=\frac{1}{t} \int_{0}^{t} \varphi(s) d s
$$

Formula (3.2) looks simple in the case of an ergodic flow. Let $h$ be the density of a probability measure on $\mathbb{R}=\{\omega\}$. Then

$$
\lim _{t \rightarrow \infty} \int_{-\infty}^{+\infty} \int_{\Lambda} h(\omega) f_{1}\left(g^{\omega t}(x)\right) f_{2}(x) d \nu d \omega=\nu(\Lambda) \int_{\Lambda} f_{1} d \nu \int_{\Lambda} f_{2} d \nu
$$

Hence, in average, the functions

$$
f_{1}\left(g^{\omega t}(x)\right) \text { and } f_{2}(x)
$$

are statistically independent for large $t$ : the integral of the product equals the product of the integrals. Particular cases of (3.3) are presented in [3].

The proof of Theorem 2 is based on the von Neumann ergodic theorem. For any $\varepsilon>0$, there exists a piecewise constant function $h_{\varepsilon}: \mathbb{R} \rightarrow \mathbb{R}$ such that

(1) $h_{\varepsilon}(\omega)=c_{k}=$ const on the intervals $\left(\omega_{k}, \omega_{k+1}\right), k=1, \ldots, N$ (the cases $\omega_{1}=-\infty$ and $\omega_{N+1}=+\infty$ are allowed);

(2) $I \subset\left(\omega_{1}, \omega_{N+1}\right)$;

(3) $\int_{I}\left|h-h_{\varepsilon}\right| d \omega<\varepsilon$.

Then, using the $g^{t}$-invariance of the measure $\nu$, we obtain

$$
\begin{gathered}
\left|\int_{I} h(\omega)\left(f_{1} \circ g^{t}, f_{2}\right) d \omega-\int_{I} h_{\varepsilon}(\omega)\left(f_{1} \circ g^{t}, f_{2}\right) d \omega\right| \\
\leq \int_{I}\left|h-h_{\varepsilon}\right| d \omega\left\|f_{1}||\right\| f_{2}|| \leq \varepsilon\left\|f_{1}\right\|\left\|f_{2}\right\|,
\end{gathered}
$$

where $\|\cdot\|$ is the $L_{2}$-norm. Hence it is sufficient to establish the convergence of the integrals

$$
J_{k}(t)=\int_{\omega_{k}}^{\omega_{k+1}} h_{\varepsilon}(\omega)\left(f_{1} \circ g^{t}, f_{2}\right) d \omega
$$

By the von Neumann ergodic theorem,

$$
J_{k}(t)=\frac{c_{k}}{t} \int_{\omega_{k} t}^{\omega_{k+1} t}\left(f_{1} \circ g^{t}, f_{2}\right) d s \rightarrow c_{k}\left(\omega_{k+1}-\omega_{k}\right)\left(\bar{f}_{1}, f_{2}\right)
$$

as $t \rightarrow \infty$. It remains to observe that

$$
\sum_{1}^{N} c_{k}\left(\omega_{k+1}-\omega_{k}\right)=\int_{I} h_{\varepsilon}(\omega) d \omega=\int_{I} h(\omega) d \omega+\delta
$$

with $|\delta| \leq \varepsilon$. 


\section{Limit MEASURES OF FOLIATED FLOWS}

In this section, we apply the method of Sec. 3 to dynamical systems with foliated phase flows. These systems are particular cases of (3.1), where $\omega$ is one-dimensional $(m=1)$. Their basic property is as follows: flows on $\Lambda$ are conjugated for different $\omega$ after a proper change of time.

Let $I \in \mathbb{R}$ be an interval (maybe infinite), and let $\Lambda$ be a smooth manifold. In the phase space $\Gamma=\Lambda \times I$, consider the dynamical system (3.1), where $z \in \Lambda$ and $\omega \in I$. We put

$$
P_{\gamma}=\{(z, \omega) \in \Gamma: \omega=\gamma\}
$$

These $n$-dimensional manifolds are invariant. The map $\psi_{\omega}:(z, \omega) \rightarrow z$ determines a natural diffeomorphism of $P_{\omega}$ and $\Lambda$. The vector field $v$ of the system (3.1) is tangent to $P_{\omega}$ at points $(z, \omega) \in \Gamma$. Let $v_{\omega}$ denote the restriction of $v$ to $P_{\omega}$.

Let $\left\{g^{t}\right\}$ be the phase flow on $\Gamma$ generated by the system (3.1), and let $\left\{g_{\omega}^{t}\right\}$ be its restriction to $P_{\omega}$. Since all the manifolds $P_{\omega}$ are diffeomorphic to $\Lambda$, we can regard $\left\{g_{\omega}^{t}\right\}$ as a one-parameter group of transformations of $\Lambda$.

Definition 4.1. We say that the flow $g^{t}$ is foliated if there exist a smooth function $\alpha: I \rightarrow(0, \infty)$ and a flow $g_{*}^{s}: \Lambda \rightarrow \Lambda$ such that the diagram

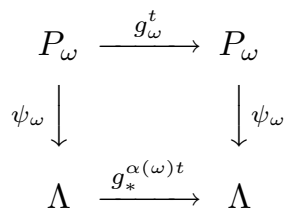

is commutative for all $\omega \in I$ and all $t \in \mathbb{R}$. We say that a foliated flow is nondegenerate if the function $\alpha(\omega)$ has only isolated critical points.

Identifying $P_{\omega}$ and $\Lambda$ by the diffeomorphism $\psi_{\omega}$, we can present the definition of a foliated flow as follows:

$$
g_{\omega}^{t}=g_{*}^{\alpha(\omega) t} .
$$

We have the following obvious result.

Proposition 4.1. Assume that the flow $g_{*}^{t}$ preserves a measure $\nu_{*}$ on $\Lambda$, and $\sigma$ is a measure on the interval $I$. Then the flow $g^{t}$ on $\Lambda \times I$ preserves the measure $\mu=\nu_{*} \times \sigma$.

Our main result is as follows.

Theorem 3. Assume that $g^{t}$ is a nondegenerate foliated flow on $\Gamma=\Lambda \times I$, the measure $\nu_{*}$ is absolutely continuous with respect to the measure determined on $\Lambda$ by some Riemannian metric, the measure $\sigma$ is absolutely continuous with respect to the Lebesgue measure on $\mathbb{R}$, and $\nu_{*}(\Lambda)=1$. Then for any $f^{\prime}, f^{\prime \prime} \in L_{2}(\Gamma, \mu)$, there exists the limit $\lim _{t \rightarrow \infty}\left(f^{\prime} \circ g^{t}, f^{\prime \prime}\right)$.

Theorems 1 and 3 imply the following corollary.

Corollary 4.1. For dynamical systems (3.1) with nondegenerate foliated phase flows, the density of the probability distribution $\rho_{t}$ has weak limits as $t \rightarrow+\infty$ and $t \rightarrow-\infty$. These limits coincide and satisfy (2.1).

Remark. Theorem 3 remains valid if $f^{\prime} \in L_{p}(\Gamma, \mu)$ and $f^{\prime \prime} \in L_{q}(\Gamma, \mu)$, where $1 / p+1 / q=1,1 \leq p \leq q \leq \infty$.

We prove Theorem 3 in the next section, and now we present some examples of systems with foliated flows.

Let $P_{\omega}, \omega>0$, be a one-parameter family of smooth manifolds, and let $\varphi_{\omega}: P_{1} \rightarrow P_{\omega}$ be a family of diffeomorphisms. The union

$$
\Gamma=\cup_{\omega>0} P_{\omega}
$$

has the structure of the cross product $\Lambda \times I$, where $\Lambda=P_{1}$ and $I=(0,+\infty)$. We endow $\Gamma$ with the smooth structure of the cross product $\Lambda \times I$.

Definition 4.2. A vector field $v$ on $\Gamma$ is called $\varphi_{\omega}$-homogeneous of degree $k$ if for some vector field $v_{1}$ on $P_{1}$,

$$
v \circ \varphi_{\omega}=\omega^{k}\left(D \varphi_{\omega}\right) v_{1}
$$

for all $\omega>0$. (Here $D$ stands for the differential.)

Since $v_{1}$ is tangent to $P_{1}$ and $\varphi_{\omega}\left(P_{1}\right)=P_{\omega}$, the field $v$ is tangent to the leaves $P_{\omega}, \omega>0$. 
Example. Let $(M,\langle\rangle$,$) be a smooth Riemannian manifold, and let \langle,\rangle^{*}$ be the metric conjugated to the metric $\langle$,$\rangle . Let p$ be an element of the conjugated space $T_{q}^{*} M$ (a momentum of the corresponding mechanical system). We put $\|p\|^{2}=\langle p, p\rangle^{*}$ and

$$
P_{s}=\left\{p \in T_{q}^{*} M: q \in M,\|p\|=s\right\}
$$

It is clear that $\Gamma=T^{*} M \backslash P_{0}$.

Let $v$ be a Hamiltonian vector field on $\Gamma$ determined by the standard symplectic structure $\sum d p_{i} \wedge d q_{i}$ and the Hamiltonian $H=\|p\|^{2} / 2$. This field generates a dynamical system on $\Gamma$, the geodesic flow. The corresponding phase flow $g^{t}$ is well defined for all $t \in \mathbb{R}$ provided that $(M,\langle\rangle$,$) is a complete Riemannian manifold (the length$ of any geodesic is infinite).

Let $(q, p)$ be a point of $\Gamma$. We put

$$
\varphi_{\omega}(q, p)=(q, \omega p), \quad \omega>0
$$

Then the vector field $v$ determined by the Hamiltonian equations

$$
\dot{q}=\partial H / \partial p, \quad \dot{p}=-\partial H / \partial q
$$

is $\varphi_{\omega}$-homogeneous of degree $k=1$. Indeed, the structure of equations (4.4) is such that $\dot{p}$ is quadratic in $p$ and $\dot{q}$ is linear in $p$.

Example. Consider a Hamiltonian system (4.4) in $\mathbb{R}^{2 n}=\left\{q_{1}, \ldots, q_{n}, p_{1}, \ldots, p_{n}\right\}$ with Hamiltonian

$$
H=\frac{1}{2} \sum_{i=1}^{n}\left(p_{i}^{2}+\alpha_{i}^{2} q_{i}^{2}\right), \quad \alpha_{i}>0
$$

(a harmonic oscillator with frequencies $\alpha_{1}, \ldots, \alpha_{n}$ ). We put again

$$
P_{\omega}=\left\{(p, q) \in \mathbb{R}^{2 n}: H(p, q)=\omega\right\}, \quad \omega>0,
$$

and $\varphi_{\omega}:(q, p) \rightarrow(\omega q, \omega p)$. The corresponding Hamiltonian vector field on $\Gamma=\cup_{\omega>0} P_{\omega}$ is $\varphi_{\omega}$-homogeneous of zero degree $(k=0)$.

Proposition 4.2. Let $v$ be a $\varphi_{\omega}$-homogeneous vector field of degree $k$ on $\Gamma$. Then $v$ generates on $\Gamma$ a foliated flow $g^{t}$, where $\alpha(\omega)=\omega^{k}$.

Corollary 4.2. For $k \neq 0$, the flow $g^{t}$ is nondegenerate.

Proof of Proposition 4.2. We put $\Lambda=P_{1}$. Note that any point $z \in \Gamma$ can be presented in the form

$$
z=\varphi_{\omega}\left(z_{1}\right), \quad \omega>0, \quad z_{1} \in P_{1}
$$

Since the map $\Lambda \times(0,+\infty) \rightarrow \Gamma$ determined by (4.5) is a diffeomorphism, it is sufficient to check that

$$
g^{t} \circ \varphi_{\omega}=\varphi_{\omega} \circ g_{1}^{\omega^{k} t}
$$

where $g_{1}^{t}$ is the restriction of the flow $g^{t}$ to $P_{1}$.

This equation obviously holds for $t=0$. Differentiating with respect to $t$ at $t=0$ yields

$$
\begin{aligned}
\left.\frac{d}{d t}\right|_{t=0} g^{t} \circ \varphi_{\omega} & =v \circ \varphi_{\omega}, \\
\left.\frac{d}{d t}\right|_{t=0} \varphi_{\omega} \circ g_{1}^{\omega^{k} t} & =\omega^{k}\left(D \varphi_{v}\right) v_{1},
\end{aligned}
$$

where $v_{1}$ is the restriction of $v$ to $P_{1}$. This equation follows from (4.3). The same equation for an arbitrary $t$ follows from the fact that $g^{t}$ is a group with respect to $t$. 


\section{Foliated flows and evolution of measures}

In this section, we prove Theorem 3. Let $f^{\prime}$ and $f^{\prime \prime}$ be functions from $L_{2}(\Gamma, \mu), \Gamma=\Lambda \times I$. We put

$$
f_{\omega}^{\prime}(\cdot)=f^{\prime}(\cdot, \omega), \quad f_{\omega}^{\prime \prime}(\cdot)=f^{\prime \prime}(\cdot, \omega) .
$$

By the Fubini theorem,

$$
\left(f^{\prime} \circ g^{t}, f^{\prime \prime}\right)=\int_{I}\left(f_{\omega}^{\prime} \circ g_{\omega}^{t}, f_{\omega}^{\prime \prime}\right) d \sigma=\int_{I}\left(f_{\omega}^{\prime} \circ g_{*}^{\alpha(\omega) t}, f_{\omega}^{\prime \prime}\right) d \sigma .
$$

Let $A^{\prime}, A^{\prime \prime} \subset \Lambda$ be measurable sets, $I^{\prime}, I^{\prime \prime}$ intervals in $I$, and $\chi^{\prime}, \chi^{\prime \prime}: \Gamma \rightarrow \mathbb{R}$ the indicators of the sets $A^{\prime} \times I^{\prime}$ and $A^{\prime \prime} \times I^{\prime \prime}$, respectively. We put

$$
J(t)=\left(\chi^{\prime} \circ g^{t}, \chi^{\prime \prime}\right)
$$

Main lemma. There exist the limits $\lim _{t \rightarrow+\infty} J(t)$ and $\lim _{t \rightarrow-\infty} J(t)$.

The main lemma implies Theorem 3. Indeed, recall that $\nu_{*}$ is absolutely continuous with respect to the measure on $\Lambda$ determined by some Riemannian metric and $\sigma$ is absolutely continuous with respect to the Lebesgue measure. Therefore the space $C_{0}^{0}(\Gamma)$ of continuous functions with compact supports is dense in $L_{2}(\Gamma, \mu)$. On the other hand, the space $\mathbf{I}(\Gamma)$ of functions that are finite linear combinations of the indicators of $\mu$-measurable sets is dense in $C_{0}^{0}(\Gamma)$ (even in the $C^{0}$-norm).

Now let $f^{\prime}$ and $f^{\prime \prime}$ be two functions from $L_{2}(\Gamma, \mu)$. To prove the existence of the limit

$$
\lim _{t \rightarrow+\infty}\left(f^{\prime} \circ g^{t}, f^{\prime \prime}\right),
$$

it is sufficient to show that given $\varepsilon>0$, the difference

$$
\left(f^{\prime} \circ g^{t_{1}}, f^{\prime \prime}\right)-\left(f^{\prime} \circ g^{t_{2}}, f^{\prime \prime}\right)
$$

is less than $\varepsilon$ for all $t_{1}, t_{2}>T(\varepsilon)$. This follows from the main lemma and the fact that the functions $f^{\prime}$ and $f^{\prime \prime}$ can be approximated with arbitrary precision by functions from $\mathbf{I}(\Gamma)$.

Now let us prove the main lemma. We put $A_{0}=A^{\prime} \cap A^{\prime \prime}$ and $I_{0}=I^{\prime} \cap I^{\prime \prime}$. Let $\tilde{\chi}_{0}: \Lambda \rightarrow \mathbb{R}$ be the indicator of $A_{0}$. It is clear that

$$
J(t)=\int_{I_{0}}\left(\widetilde{\chi}_{0} \circ g_{*}^{\alpha(\omega) t}, \tilde{\chi}_{0}\right) d \sigma .
$$

Let $D_{\gamma}=\left\{\omega \in I_{0}:\left|\alpha^{\prime}(\omega)\right|>\gamma\right\}, \alpha^{\prime}=d \alpha / d \omega$. Since the critical points of the function $\alpha(\omega)$ are isolated, the $\sigma$-measure of the set $I \backslash D_{\gamma}$ tends to zero as $\gamma \rightarrow 0$. Furthermore, we may regard $D_{\gamma}$ as the union of a finite set of intervals. Let $\left(\omega_{1}, \omega_{2}\right)$ be one of these intervals. Then $\alpha$ can be regarded as a coordinate on $\left(\omega_{1}, \omega_{2}\right)$. Indeed, there exists a smooth function $\omega(\alpha)$ inverse to $\alpha:\left(\omega_{1}, \omega_{2}\right) \rightarrow \mathbb{R}$. We put $d \sigma(\omega)=h(\omega) d \omega$. According to the conditions of Theorem 3, the function $h(\omega)$ is integrable: $h \in L_{1}(I, d \omega)$. Hence

$$
\int_{\omega_{1}}^{\omega_{2}}\left(\widetilde{\chi}_{0} \circ g_{*}^{\alpha(\omega) t}, \tilde{\chi}_{0}\right) h(\omega) d \omega=\int_{\alpha\left(\omega_{1}\right)}^{\alpha\left(\omega_{2}\right)}\left(\tilde{\chi}_{0} \circ g_{*}^{\alpha t}, \tilde{\chi}_{0}\right) h(\omega(\alpha)) \omega^{\prime}(\alpha) d \alpha .
$$

Since $h(\omega(\alpha)) \omega^{\prime}(\alpha) \in L_{1}\left(\left(\alpha\left(\omega_{2}\right), \alpha\left(\omega_{1}\right)\right), d \alpha\right)$, we see that, by Theorem 2, the integral (5.2) has a limit as $t \rightarrow \infty$.

\section{INCREASE OF THE ENTROPY}

The entropy is defined in statistical mechanics as the integral

$$
S_{t}=-\int_{\Gamma} \rho_{t} \log \rho_{t} d \mu
$$

Since $\rho_{t}(z)=\rho \circ g^{-t}(z)$ and the flow $g^{t}$ preserves the measure $\mu$, we have $S_{t}=$ const. This remark is a particular case of the Poincaré result (see [2]).

On the other hand, $\rho_{t}$ weakly converges to $\bar{\rho}$ as $t \rightarrow \pm \infty$. Following Gibbs and Poincaré, we can say that the stationary density of the probability distribution $\bar{\rho}$ corresponds to a heat equilibrium of the system in question. Thus it is natural to introduce the entropy of the equilibrium state:

$$
S_{\infty}=-\int_{\Gamma} \bar{\rho} \log \bar{\rho} d \mu
$$




\section{Theorem 4.}

$$
S_{t} \leq S_{\infty}
$$

The proof is based on the fact that the function $h(x)=-x \log x$ is concave for positive $x$. Since $S_{t}=$ const, by the Fubini theorem,

$$
S_{t}=\frac{1}{T} \int_{0}^{T} \int_{\Gamma} h\left(\rho_{t}\right) d \mu d t=\int_{\Gamma}\left[\frac{1}{T} \int_{0}^{T} h\left(\rho_{t}\right) d t\right] d \mu .
$$

By the Jensen inequality,

$$
\frac{1}{T} \int_{0}^{T} h\left(\rho_{t}\right) d t \leq h\left(\frac{1}{T} \int_{0}^{T} \rho_{t} d t\right), \quad T>0 .
$$

Therefore

$$
S_{t} \leq \int_{\Gamma} h\left(\frac{1}{T} \int_{0}^{T} \rho_{t} d t\right) d \mu
$$

It remains to consider the $T \rightarrow \infty$ limit and use Theorem 1 .

Remark. For a medium without collisions in a right parallelepiped, inequality (6.1) is established in [5]. Some cases of this inequality are presented in $[2$, item 6$]$.

Since $\rho_{t}$ weakly converges to $\bar{\rho}$ as $t \rightarrow+\infty$ and $t \rightarrow-\infty$, the assertion of Theorem 4 concerning the entropy increase is invariant with respect to the time reversal. Recall that the kinetic Boltzmann equation implies monotone entropy growth as the time increases.

Acknowledgments. The authors thank V. Ten for useful comments.

The work is partially supported by INTAS grant 00-221 and RFBR grants 02-01-00400 and 00-15-99269.

\section{REFERENCES}

1. W. Gibbs, Elementary Principles in Statistical Mechanics Developed with Especial Reference to the Rational Foundation of Thermodynamics, New York (1902).

2. H. Poincaré, "Réflections sur la théorie cinétique des gaz," J. Phys. Théoret. et Appl., 4e Sér., 5, 369-403 (1906).

3. V. V. Kozlov, "Heat equilibrium according to Gibbs and Poincaré," Dokl. Akad. Nauk, 382, No. 5, 602-606 (2002).

4. V. V. Nemytsky and V. V. Stepanov, Qualitative Theory of Differential Equations [in Russian], Moscow (1947).

5. V. V. Kozlov, "Kinetic of collisionless continuous medium," Regul. Chaotic Dyn., 6, No. 3, 235-251 (2001). 